\title{
PP20
}

\section{Assessing the Proportions and CCT of Direct//ndirect Lighting in a Real Lit Office}

Yan Lu et al.

DOI 10.25039/x46.2019.PP20

from

CIE x046:2019

Proceedings

of the

29th CIE SESSION

Washington D.C., USA, June 14 - 22, 2019

(DOI 10.25039/x46.2019)

The paper has been presented at the 29th CIE Session, Washington D.C., USA, June 14-22, 2019. It has not been peer-reviewed by CIE.

(C) CIE 2019

All rights reserved. Unless otherwise specified, no part of this publication may be reproduced or utilized in any form or by any means, electronic or mechanical, including photocopying and microfilm, without permission in writing from CIE Central Bureau at the address below. Any mention of organizations or products does not imply endorsement by the CIE.

This paper is made available open access for individual use. However, in all other cases all rights are reserved unless explicit permission is sought from and given by the CIE.

CIE Central Bureau

Babenbergerstrasse 9

A-1010 Vienna

Austria

Tel.: +4317143187

e-mail: ciecb@cie.co.at

www.cie.co.at 


\title{
ASSESSING THE PROPORTIONS AND CCT OF DIRECT/INDIRECT LIGHTING IN A REAL LIT OFFICE
}

\author{
Lu, Y..$^{1,2}$, Hou, D. ${ }^{1,2}$, Lin, Y..$^{1,2}$ \\ ${ }^{1}$ Institute for Electric Light Sources, Fudan University, Shanghai, CHINA \\ ${ }^{2}$ Engineering Research Center of Advanced Lighting Technology, Ministry of Education, \\ Shanghai, CHINA \\ ydlin@fudan.edu.cn
}

DOI 10.25039/x46.2019.PP20

\begin{abstract}
This paper explores how the proportions and CCT of direct/indirect lighting in office affect the subjective perception and work performance of people. Two parts of psychophysical experiments were conducted in a real lit office adjusting the illuminance and CCT of uplight/downlight, respectively. Observers were asked to rate subjective evaluation and test cognitive performance under different light settings. The results of this study showed that both photometric and colorimetric distributions of office lighting influence subject evaluation. Office lighting supplemented by indirect lighting with consistent or inconsistent CCT will improve spaciousness of the room and preference for the environment. However, neither the illuminance nor CCT distribution of the space affects work performance as long as the illuminance and CCT at work plane are held constantly.
\end{abstract}

Keywords: Direct/Indirect light, illuminance, CCT, Subjective Evaluation, office lighting

\section{1. Introduction}

There is ample evidence to support the hypothesis that indoor lighting environment has impact on human affective and cognitive processes. Light distribution is one of the important aspects of the indoor lighting environment. With the development of various pendant luminaires and LED technology, both photometric and colorimetric distribution in vertical can be adjustable thus different lighting schemes can be achieved to satisfy the need of lighting design.

Laboratory studies have shown that people tend to prefer lighting systems with indirect lighting components to systems providing just direct lighting and the ratio of direct/indirect lighting can modulate spacious perception(Harvey, 1984, Robert Yearout, 1989). Yu-bin Shin's study that indirect lighting makes people feel happy and awake through physiological parameter analysis and subjective evaluation analysis(Shin et al., 2015). However, there are some inconsistent conclusions from previous studies and not all of them support mixed lighting. A field study by Collins et al. further shows that indirect lighting system was less preferred to other mixed lighting system(Belinda L. Collins, 1990). A Norwegian study proposed that the light distribution ratio of $75 \%$ indirect lighting and $25 \%$ direct lighting could improve the lighting environment and visual conditions sustainably (Arne Aarås, 2001). In contrast, Wolska revealed increased eye fatigue with mixed lighting(Wolska, 2003). KI Fostervolda believes that the proportion of light distribution has no obvious influence on people's work performance in a one-year intervention experiment(Fostervold and Nersveen, 2008). Besides, since these researches focused on the illuminance distribution of direct/indirect lighting system, there is a lack of study considering the colorimetric distribution of mixed lighting. Will different CCT of uplight and downlight affect occupants' assessment and behaviour to the lighting environment?

As an important occasion of work, offices of different scale have got the most attention from different researches. For the lighting environment in office space, both occupants' feeling and evaluation and visual behaviour matter. The present study of light distribution in a real lit office conducted two parts of experiments. The aim is to discuss the impact of proportions of direct/indirect lighting on both subjective evaluation and work performance, and also include 
the discussion of the distribution of correlated color temperature (CCT) of uplight/downlight in an office space.

\section{Methods}

\subsection{Subjects}

15 Chinese participants aged from 21 to 30 years (mean age $=23.4$ years, $S D=2.77$ years) were recruited for this experiment, including 7 males and 8 females. The Ishihara test was used for to examine vision and all participants had normal vision.

\subsection{Experiment setup}

The experiments were carried out to study the distribution of illuminance and colour temperature in a real lit office. The room had a size of $3.2 \mathrm{~m} \times 3 \mathrm{~m} \times 2.8 \mathrm{~m}$. Six pendant fixtures were installed and the brightness and CCT of uplight/downlight could be controlled respectively. Table 1 shows the Illuminance proportions and CCT of uplight/downlight. The experiments were separated into two parts. In the first part, the proportions of direct/indirect lighting (Light setting 1-5) changed. Lighting parameters including illuminance and CCT on working plane were held constantly $(6000 \pm 100 \mathrm{~K}, 300 \pm 5 \mathrm{Ix})$. In the second part, the CCT of uplight/downlight changed (Light setting 6-8) and the CCT and illuminance on working plane were also kept in constant, which are $4800 \pm 100 \mathrm{~K}$ and $300 \pm 10 \mathrm{~lx}$ for all these three light settings. Figure 1 shows the experimental environment.

Table 1 - Lighting parameters of each light setting

\begin{tabular}{|c|c|c|c|c|c|c|c|c|c|}
\hline Light & Setting & 1 & 2 & 3 & 4 & 5 & 6 & 7 & 8 \\
\hline $\begin{array}{l}\text { Illuminance } \\
\text { proportions }\end{array}$ & uplight & 100 & 75 & 50 & 25 & 0 & 50 & 50 & 50 \\
\hline $\begin{array}{c}\text { proportions } \\
(\%)\end{array}$ & downlight & 0 & 25 & 50 & 75 & 100 & 50 & 50 & 50 \\
\hline \multirow{2}{*}{ CCT (K) } & uplight & 6000 & 6000 & 6000 & 6000 & 6000 & 6000 & 3800 & 3000 \\
\hline & downlight & 6000 & 6000 & 6000 & 6000 & 6000 & 4000 & 6500 & 8600 \\
\hline
\end{tabular}



Figure 1 - Photographs showing lighting settings of proportions (top row) and CCT distributions (bottom row) of direct/indirect light 


\subsection{Psychophysical experiment}

In the first part of experiment, each observer viewed the test lighting conditions following a random order. In the second part of experiment, the three light settings were viewed in three sessions. Each session was separated by 2 days due to the time setting up the experimental light. Observers were asked to assess the same direct lighting condition (6000K, 300lx on work plane) in every session to test the baseline of each observer in different experimental days.

For each light setting, observers were asked to assess the lighting environment in terms of subjective evaluation, work performance and fatigue. Subjective impressions were evaluated using 7-point categorical judgment methods. Scales in previous study were modified considering 7 parts of content: subjective brightness, objective evaluation, visual comfort, uniformity, spaciousness, readability and preference, as listed in table 2(Houser et al., 2002). For work performance assessment in office, the d2 Test of Attention (D2), a cancellation test, and the Paced Visual Serial Addition Task (PVSAT) are used as visual tasks. The D2 assesses performance in terms of visual perceptual speed and concentrative capacities by assessing an individual's ability to selectively. The Paced Visual Serial Addition Task (PVSAT) is an addition task heavily dependent on frontal brain regions, which involves higher order executive functioning. Besides, critical fusion frequency (CFF) and questionnaires were used to reflect fatigue in different light settings. CFF values were measured before and after each task session while questionnaires were used after visual tasks.

Table 2 - The subjective evaluated scales studied in seven categories

\begin{tabular}{ll}
\hline $\begin{array}{l}\text { subjective } \\
\text { brightness }\end{array}$ & $\begin{array}{l}\text { Bight (ceiling, desktop, walls, floor, room } \\
\text { overall) } \\
\text { Harsh/mild shadow, dull/sharp edges, } \\
\text { objective evaluation } \\
\text { attractive } \\
\text { visual comfort }\end{array}$ \\
$\begin{array}{l}\text { Comfort, glare, light quality } \\
\text { Uniformity }\end{array}$ & overall) \\
spaciousness & Large, spacious \\
readability & Clear, recognizable (while reading), \\
preference & Like, pleasant, satisfied, energetic \\
\hline
\end{tabular}

\section{Results and discussion}

\subsection{Statistical analysis}

Data were analyzed in the Statistical Package for Social Science version 22.0 (SPSS Inc., Chicago, IL). The alpha level was set at 0.05 for all statistical tests, and all p-values were two tailed. Analysis of variance(ANOVA) were made to analysis people's assessments of the distribution of illuminance and colour temperature of office lighting. Multiple comparison groupings based on LSD method were also used to isolate which of the differences between sets of means contributes to the overall difference.

\section{2 subjective evaluation}

For the first part of experiment, the results of subjective brightness show a significant downward trend on perceived brightness of ceiling when the proportions of indirect decrease $(p=0.000)$. The brightness of wall shows a small downward trend when room is lit more direct but the noticeable difference only shows between light setting $1 \& 4$ and $1 \& 5$, which means noticeable wall's brightness change need large difference. Bivariate correlation analysis shows there are significant linear correlations between overall brightness and brightness of wall (Pearson's $r=0.822$ ), floor (Pearson's $r=0.711$ ), desk (Pearson's $r=0.711$ ), and ceiling (Pearson's $r=0.475$ ). The perceived overall brightness of the experimental office room can be described as the following equation: 


$$
B_{\text {overall }}=0.25+0.357 B_{\text {wall }}+0.241 B_{\text {floor }}+0.282 B_{\text {desk }}+0.103 B_{\text {ceiling }}
$$

The overall brightness trend shows similar results with previous study but the correlation results between different scale are different, which indicates brightness perception is related to the specific office space.

According to the subjective ratings, light settings with more indirect light and less direct light also lead to milder shadows of objects $(p=0.000)$, more eye's comfort $(p=0.046)$, more feelings of spaciousness $(p=0.011)$ and pleasure $(p=0.008)$. Observers also express more preference when more light was upward $(p=0.002)$. However, people tend to feel tiredness easily in lighting environment contains more indirect component $(p=0.028)$.

The uniformity of ceiling, wall, floor, and the overall room changes significantly when light changes and the uniformity except for the floor's reaches the highest in the light setting 3 (direct/indirect light). The floor uniformity decreases significantly $(p=0.032)$ when more down light are supplied, which may be caused by the harsh shadow of the objects on floor such as desk and chair.

For the CCT distribution of uplight/downlight, there is significant decrease in perceived walls' uniformity when the difference of CCT between uplight and downlight becomes larger $(p=0.031)$.

The CCT distribution seems do not affect the perceived brightness of different part of office. Bivariate correlation analysis also shows significant linear correlations between overall brightness and brightness of wall (Pearson's r=0.706), floor (Pearson's r=0.620), desk (Pearson's $r=0.696$ ), and ceiling (Pearson's $r=0.398$ ). The correlation between brightness perception results here and the linear regression model in the first part was tested and the $r^{2}=0.80$. This shows that the linear regression model has a relatively good fitting degree when light settings changes, which may offer some solutions to modify the space brightness with light distribution.

For other scales used in experiment, different observer seems to have different preference or feeling about these three light settings. No obvious relationships are found between CCT distribution and other subjective evaluation results.

Compared all the light settings in view of the same baseline, conditions with indirect components improved spaciousness of the room and preference for the environment. Overall, direct lighting supplemented by indirect lighting with consistent or inconsistent CCT is superior to direct lighting in the office in terms of subjective evaluation.

\section{3 cognitive performance and fatigue}

Table 3 and Table 4 shows the results of the D2 test and the PVSAT test. One-way ANOVA for repeated measurements was conducted. The results for these two cognitive performance shows no significant difference between the five light settings (1-5) with different proportions of uplight/downlight and between the three light settings (6-8) with different CCT distributions as well. No effect of light settings on fatigue was found either.

Table 3 - Test results of the D2 test (Mean and standard deviation)

\begin{tabular}{lllllllll}
\hline Measure & 1 & 2 & 3 & 4 & 5 & 6 & 7 & 8 \\
\hline TN & $607.5(40.0)$ & $612.9(39.8)$ & $592.4(58.0)$ & $608.5(46.6)$ & $603.9(45.4)$ & $618.0(40.1)$ & $629.5(37.7)$ & $627.0(35.5)$ \\
E1 & $12.5(16.8)$ & $11.9(9.4)$ & $12.6(12.9)$ & $15.6(20.5)$ & $12.9(13.1)$ & $9.0(10.6)$ & $4.9(6.3)$ & $7.5(9.0)$ \\
E2 & $1.4(2.9)$ & $1.1(1.3)$ & $1.6(1.6)$ & $1.1(1.6)$ & $1.7(1.8)$ & $2.2(2.3)$ & $1.8(2.6)$ & $1.9(2.1)$ \\
E & $13.9(19.7)$ & $12.9(10.6)$ & $14.2(14.5)$ & $16.7(22.1)$ & $14.7(14.9)$ & $11.2(12.9)$ & $6.7(8.9)$ & $9.5(11.1)$ \\
TN-E & $593.5(47.0)$ & $599.9(43.0)$ & $578.2(63.7)$ & $591.9(56.9)$ & $589.3(49.7)$ & $606.8(47.8)$ & $622.8(42.3)$ & $617.5(38.2)$ \\
CP & $304.2(4.9)$ & $305.4(3.87)$ & $305.5(3.9)$ & $306.1(2.5)$ & $305.1(3.23)$ & $305.8(2.3)$ & $306.2(2.6)$ & $306.1(2.1)$ \\
FR & $6.1(3.3)$ & $6.7(5.3)$ & $6.4(4.7)$ & $5.5(3.4)$ & $6.1(4.0)$ & $5.9(4.2)$ & $5.3(5.6)$ & $4.1(4.0)$ \\
\hline
\end{tabular}


Notes: $\mathrm{TN}=$ total number; $\mathrm{E} 1=$ Omissions; $\mathrm{E} 2=$ Commissions; $\mathrm{E}=$ errors; $\mathrm{N}-\mathrm{E}=$ total-errors; $\mathrm{CP}=$ concentrations performance; $F R$ = fluctuation rate.

Table 4 - Test results of the PVSAT test (Mean and standard deviation)

\begin{tabular}{lllllllll}
\hline Measure & 1 & 2 & 3 & 4 & 5 & 6 & 7 & 8 \\
\hline Time/s & $142.6(12.9)$ & $143.3(17.9)$ & $151.7(19.5)$ & $144.5(14.9)$ & $142.1(11.5)$ & $140.9(15.1)$ & $131.8(7.5)$ & $134.5(9.9)$ \\
Accuracy/\% & $98.9(2.7)$ & $98.0(3.73)$ & $96.7(5.77)$ & $98.0(5.2)$ & $98.0(3.3)$ & $98.9(2.1)$ & $98.7(2.5)$ & $98.9(1.6)$ \\
Time/Accuracy & $7.0(0.7)$ & $7.0(1.0)$ & $6.5(1.0)$ & $6.9(1.0)$ & $7.0(0.7)$ & $7.1(0.7)$ & $7.5(0.5)$ & $7.4(0.6)$ \\
$10^{\wedge}-3$ & & & & & & & & \\
\hline
\end{tabular}

These results are partly consistent with KI Fostervold's study which revealed that change of the proportions of indirect and direct light in a long time intervention (half or one year) didn't affect health, well-being and cognitive performance. Our experiments indicate that as long as the illuminance and CCT at work plane are held constantly, neither the illuminance nor CCT distribution of the space can influence the work performance or fatigue of office workers'.

\section{Conclusions}

Experiments were conducted to assess perception and cognitive performance under a series light settings with different proportions and CCT of direct/indirect lighting in office. From the results above, the following conclusions can be drawn:

- It is worth considering the photometric and colorimetric distribution of office lighting because occupants are able to discriminate the differences.

- Ambient light in an indoor environment can significantly influence subject evaluation in many aspects especially the brightness and uniformity perception of different part of the room. Supplementing indirect lighting will improve spaciousness of the room and preference for the environment.

- Neither the illuminance nor CCT distribution of the space influences work performance or fatigue of office workers as long as the illuminance and CCT at work plane are held constantly, which may offer more freedom to office lighting designers.

\section{Acknowledgments}

The authors disclosed receipt of the following financial support for the research, authorship, and/or publication of this article: This research was supported by National Key R\&D Program of China (Project No.2017YFB0403700).

\section{References:}

ARNE AARÅS, G. H. H. B. (2001), "Musculoskeletal, visual and psychosocial stress in VDU operators before and after multidisciplinaryergonomic interventions. A 6 years prospective studyFPart II", Applied Ergonomics, Vol. 32 No. 6, pp. 559-571.

BELINDA L. COLLINS, W. F. G. G. (1990), "Second-Level Post-Occupancy Evaluation Analysis", Journal of the Illuminating Engineering Society, Vol. 19 No. 2, pp. 21-44.

FOSTERVOLD, K. I. \& NERSVEEN, J. (2008), "Proportions of direct and indirect indoor lighting - The effect on health, well-being and cognitive performance of office workers", Lighting Research \& Technology, Vol. 40 No. 3, pp. 175-200.

HARVEY, L. O. D. D. (1984), "Quantifying Reactions of Visual Display Operators to Indirect Lighting ", Journal of the Illuminating Engineering Society, Vol. 14 No. 1, pp. 515-546.

HOUSER, K. W., TILLER, D. K., BERNECKER, C. A. \& MISTRICK, R. G. (2002), "The subjective response to linear fluorescent direct/indirect lighting systems", Lighting Research \& Technology, Vol. 34 No. 3, pp. 243-260. 
ROBERT YEAROUT, S. K. (1989), "Visual display unit workstation lighting", International Journal of Industrial Ergonomics, Vol. 3 No. 3, pp. 265-273.

SHIN, Y., WOO, S., KIM, D., KIM, J., KIM, J. \& PARK, J. Y. (2015), "The effect on emotions and brain activity by the direct/indirect lighting in the residential environment", Neuroscience Letters, Vol. 58428-32.

WOLSKA, A. (2003), "Visual Strain and Lighting Preferences of VDT Users Under Different Lighting Systems", International Journal of Occupational Safety and Ergonomics, Vol. 9 No. 4, pp. 431-440. 